\title{
Comparative Study of Energy Harvesting from ZnO Nanorods Using Different Flexible Substrates
}

Mushtaque Hussain, Mazhar Ali Abbasi, Azam Khan, Omer Nur and Magnus Willander

\author{
Linköping University Post Print
}

Tweet

N.B.: When citing this work, cite the original article.

Original Publication:

Mushtaque Hussain, Mazhar Ali Abbasi, Azam Khan, Omer Nur and Magnus Willander, Comparative Study of Energy Harvesting from ZnO Nanorods Using Different Flexible Substrates, 2014, Energy Harvesting and Systems, (1), 1-2, 19-26.

http://dx.doi.org/10.1515/ehs-2013-0025

Copyright: De Gruyter; Energy Harvesting and Systems http://www.degruyter.com/

Postprint available at: Linköping University Electronic Press http://urn.kb.se/resolve?urn=urn:nbn:se:liu:diva-108226 


\title{
Comparative study of energy harvesting from $\mathrm{ZnO}$ nanorods using different flexible substrates
}

\author{
Mushtaque Hussain, Mazhar Ali Abbasi, Azam Khan, Omer Nur, Magnus Willander \\ Department of Science and Technology, Campus Norrköping, Linköping University, SE- \\ 60174 Norrköping, Sweden.
}

Correspondening author: mushatque.hussain@liu.se

Phone number: +46-11-363441; Fax: +46-11-363270

\begin{abstract}
The step toward the fabrication of nanodevices with low cost and improved performance is of high demand; therefore, in the present study, different flexible

substrates like common paper, textile fabric, plastic and aluminum foil have been utilized to harvest electrical energy. $\mathrm{ZnO}$ nanorods (NRs) were grown by using low temperature aqueous chemical growth method. The obtained $\mathrm{ZnO}$ NRs were highly dense, well aligned, uniformly distributed over the substrates and exhibited good crystal quality. The structural study was carried out by using X-ray powder diffraction and scanning electron microscopy. The piezoelectric properties of $\mathrm{ZnO}$ NRs were investigated by the help of an atomic force microscope using contact mode. The measurements of generated piezoelectricity were around $16.2 \mathrm{mV}, 23.2 \mathrm{mV}, 38.5 \mathrm{mV}$ and $43.3 \mathrm{mV}$ for common paper, textile fabric, plastic and aluminum foil, respectively. This investigation is an important step in order to study the effect of different substrates influencing the magnitude of the output voltage under identical growth and measurement conditions. We expect that this study will help identify the most suitable flexible substrate for harvesting energy. It also offers a promising alternative powering source for the next generation nanodevices using non-conventional substrates like aluminum foil. Moreover, the use of aluminum foil as flexible and low cost substrate may pave the way to develop devices in different fields including energy harvesting.
\end{abstract}

Keywords: $\mathrm{ZnO}$ nanorods, flexible substrates, aqueous chemical growth, atomic force microscope. 


\section{Introduction:}

With the enormous use of energy resources at large scale, the threat of global warming and energy crisis is increasing day by day. To overcome these issues, exploring alternative energy resources is desperately needed, especially focusing on renewable and green energy (environment friendly) resources. In order to overcome this challenge, the development is taking place both at large and at small scales. Researchers and scientists are working hard for independent, sustainable and maintenance free energy, which can be utilized to operate small devices like mobile phones, implantable biosensors, ultrasensitive chemical and biomolecular sensors, nanorobotics, remote and mobile environmental sensors and even portable/wearable personal electronics. Therefore, it is highly desirable to develop self-power nanodevices, which can harvest energy from the environment. One example is the use of one-dimensional (1D) forms of inorganic materials in flexible and transparent electronics (Bradley, Gabriel, and Gruner 2003; Cao et al. 2006). As the 1D materials provide a significant enhancement in the mechanical flexibility and optical transparency, while their single crystalline structures hold promise for high device performance. Using micro/nanofabrication techniques, various types of devices with good performance have been demonstrated, including light-emitting diodes (Park and Yi 2004), UV nanolasers (Huang et al. 2001), UV photo detectors (Soci et al. 2007), field-effect transistors (Arnold et al. 2003), piezoelectric transducer/actuator (Buchine et al. 2006) and nanogenerators (Wang and Song 2006).

Zinc oxide $(\mathrm{ZnO})$ is one of the most studied II-VI group semiconductors, with a direct wide band gap (3.37 eV), a relatively large exciton binding energy (60 meV) (Sun et al. 2008) and a high electron mobility $[\sim 1,000 \mathrm{~cm} 2 /(\mathrm{V} \mathrm{s})]$ (Park et al. 2004) for a single nanowire at room temperature, which is favorable for efficient electron transport and reduced recombination loss. Keeping these properties along with biosafe and biocompatible properties, $\mathrm{ZnO}$ nanomaterials have found wide applications in various fields like optics 
(Tena-Zaera, Elias, and Levy-Clement 2008), electrical transport (Lu et al. 2011), photoconductivity (Law and Thong 2006) and especially in harvesting piezoelectricity (Wang and Song 2006). Because the $\mathrm{ZnO}$ nanostructure (NS) has a hexagonal wurtzite crystal structure, which naturally produces a piezoelectric effect once the material is strained, which resulted a built-in polarization in $\mathrm{ZnO}$ NRs along its c-axis. Therefore, by utilizing the semiconducting and piezoelectric properties of $\mathrm{ZnO}$, nanogenerators (Wang and Song 2006), piezoelectric field-effect transistors (Khan et al. 2012), piezoelectric diodes (He et al. 2007) and piezoelectric chemical sensors (Lao et al. 2007) have already been fabricated. Among these, the fabrication of nanogenerators is gaining considerable attention from researchers to fulfill the demands of alternative, cheap and green energy resources. Many reports have been already published on the fabrication of nanogenerators for harvesting piezoelectricity (Wang and Song 2006; Yang et al. 2009). The basic purpose of the development of nanogenerators is to convert the available form of mechanical energy from the environment into electric energy (Wang and Song 2006; Wang et al. 2007), so it would facilitate the development of nanodevices. Such nanogenerators can also be used to develop a battery-less system for future applications.

In recent years, various articles have been published on the piezoelectric properties of $\mathrm{ZnO}$, but most of them have focused on hard substrates like GaN, glass, sapphire, ITO or Si (Wang and Song 2006; Wang et al. 2007; Hu et al. 2010; Chang et al. 2011; Zhang, Liu, and Wang 2011). But one should keep in mind that, it is quite difficult to enhance the piezoelectric effect by transferring the mechanical energy into electrical energy using hard substrates (Hsu and Chen 2012). Therefore, researchers are now trying to exploit the piezoelectric effect of various conventional and non-conventional flexible substrates, such as textile fabric, common paper and flexible plastic (Khan et al. 2012; Qiu et al. 2012; Gao et al. 2007). However, these substrates require a conductive adhesive layer prior to 
growth. In addition to this, for paper and textile, an insulating layer is also needed to reduce the surface roughness. This study eliminates the use of insulating/conductive layer using aluminum foil as substrate, which is not only flexible and low cost but can be formed into large-area substrates. Furthermore, in this study, textile fabric, common paper, flexible plastic and aluminum foil all have been kept under identical conditions both for growth and for measurement of piezoelectricity in order to analyze the effect of substrate on magnitude of the output voltage. In addition to this, the obtained results have also been compared with the reported results.

Although $\mathrm{ZnO} \mathrm{NSs}$ can be synthesized by various techniques, including chemical vapor deposition (CVD) (Wang et al. 2007), template-assisted growth (Chik et al. 2004), metal-organic vapor phase epitaxy (Ogata et al. 2003), metal-organic CVD (Yang et al. 2004), selfcatalyzed vapor liquid solid method (Hsueh et al. 2007), sol-gel synthesis (Gullapalli et al. 2010) and low-temperature hydrothermal methods (Soci et al. 2007; Yang et al. 2009), the low-temperature aqueous chemical growth (ACG) method is better than other methods, because the growth temperature is very low, making the method suited to the chemical properties of the used flexible substrates and eliminating the problems concerning the high temperature. Furthermore, the ACG method is simple, low cost and can be adopted in mass production. One-dimensional $\mathrm{ZnO} \mathrm{NRs}$ with larger aspect (length-to-diameter) and surface-to-volume ratios than $\mathrm{ZnO}$ bulk and films are well known to have better physical and chemical properties. Energy harvesting has been shown herein using ZnO NRs on different flexible substrates including textile fabric, common paper, flexible plastic and aluminum foil. Details of the growth of $\mathrm{ZnO}$ NRs and the characteristics of the energy harvesting are also discussed. 


\section{Experimental:}

Hexamethylenetetramine [C6H12N4] and zinc nitrate hexahydrate [Zn (NO3)2 $6 \mathrm{H} 2 \mathrm{O}]$ along with aluminum foil were purchased from Sigma-Aldrich, Sweden were used without any further purification. ACG method has been employed for the growth of $\mathrm{ZnO} \mathrm{NRs}$ on all flexible substrates. First of all, a piece $(10 \mathrm{~mm} \times 10 \mathrm{~mm})$ each of textile fabric, common paper, flexible plastic and aluminum foil substrates were dipped in isopropanol in a beaker and left for few minutes. Then, these substrates were cleaned with acetone and dried with flow of nitrogen gas at room temperature. Then, a thin layer of silver (Ag) was deposited on textile fabric, common paper and flexible plastic by thermal evaporation method in order to use as a bottom electrode during the measurements. After that all the substrates were cleaned again by repeating the same process as explained above. Finally, a seed solution of zinc acetate dihydrate was spun coated 2-3 times at 2,500 rpm on all the substrates. Usually, the seed layer acts as the nucleation site, so in order to obtain uniform and well-aligned NRs, the use of the seed layer is very important. An equimolar precursor solution of zinc nitrate and hexamethylenetetramine was prepared in $200 \mathrm{~mL}$ of deionized water and was equally divided in four beakers. The seed layer containing substrates were vertically fixed in Teflon sample holder, and finally we placed the sample holders inside the beakers containing the precursor solutions and covered the beakers by aluminum foil. Then, all the beakers were kept in the preheated electric oven at $95^{\circ} \mathrm{C}$ for 5-6 h. After the completion of growth time, the substrates were taken out and washed with the deionized water in order to remove the unwanted residual particles from the surface of grown $\mathrm{ZnO}$ NRs.

An atomic force microscope (AFM) in contact mode has been used for all the scan measurements in order to realize the piezoelectric generation from $\mathrm{ZnO}$ NRs, whereas the fast scan direction was perpendicular to the cantilever $\left(0^{\circ}\right)$. Initially, the cantilever 
deflection signal was adjusted to zero with undistracted cantilever. During the measurements, the potential was set in the range of $1-5 \mathrm{~V}$. The deflection signal showed a sensitivity of 100 $\mathrm{nm} / \mathrm{V} \pm 20 \%$, while $0.6 \mathrm{~N} / \mathrm{m}$ was the spring constant of the tip producing a vertical force in between 0.6 and $3 \mu \mathrm{N}$. The cantilever was plugged into the instrument ground, and the piezoelectric current was measured by the help of an un-integrated sample holder. The gain of the trans-impedance amplifier showed by the feedback resistor was $1 \mathrm{~V} / \mu \mathrm{A}$. The positive output voltage suggested that current is flowing from tip to the $\mathrm{ZnO} \mathrm{NRs}$ in the entire circuit. In order to minimize the stray leakage current as much as possible, the feedback loop of AD822 operational amplifier was utilized to keep the sample at ground potential. Since input bias current recorded by the amplifier was less than $25 \mathrm{pA}$, it was disregarded. In order to nullify the effect of any leakage current, the gain bandwidth product of AD822 operational amplifier was about $1.8 \mathrm{MHz}$, which resulted effective impedance to ground of $500 \Omega$ at 1 $\mathrm{kHz}$ with $1 \mathrm{M} \Omega$ feedback resistor. The sample was set at less than $0.5 \mathrm{mV}$ bias voltage (bias in less than $1 \mathrm{mV}$ ). The schematic diagram in Figure 1 summarizes the explanation given above.

Before starting the measurements, few steps were taken carefully in order to rule out possible artifacts in the measurements of piezoelectric potential. Because many factors can influence the experimental results and produce artifacts, for example, error in AFM measurement setup or the coupling of the ZnO NRs with the measurement system. Therefore, to achieve reliable results with accuracy, few cautions have been applied. First of all, ZnO NR must develop a Schottky contact at one of its ends for outputting electricity. Secondly, the output voltage and current of $\mathrm{ZnO} \mathrm{NR}$ must satisfy the switching polarity and finally, the output current and voltage of multiple $\mathrm{ZnO}$ NRs must satisfy the linear superposition rule, if they are connected in parallel and series, respectively. As soon as we have all these factors in control then started to record the measurements. 
The grown $\mathrm{ZnO}$ NRs were characterized by various techniques such as field emission scanning electron microscopy using a LEO 1550 Gemini microscope at $15 \mathrm{kV}$ to investigate the morphology of the $\mathrm{ZnO} \mathrm{NRs}$. The crystal quality of as-grown $\mathrm{ZnO}$ NRs was studied by X-ray powder diffraction (XRD) using a Phillips PW 1729 powder diffractometer equipped with $\mathrm{CuK} \alpha$ radiation $(\lambda=1.5418 \AA$ ) using generator voltage of $40 \mathrm{kV}$ and current of $40 \mathrm{~mA}$. For the analysis of piezoelectricity generation from the $\mathrm{ZnO} \mathrm{NRs}$, the measurements were performed using an AFM (Digital Instruments Multimode AFM, Netherlands) and a custom-made trans-impedance amplifier and stiff platinum-coated probes (NT-MDT NSG01/Pt, Russian Federation).

\section{Results and discussion:}

Figure 2(a-d) shows the SEM images of ZnO NRs grown on different substrates including common paper, flexible plastic, textile fabric and aluminum foil, respectively. It can be seen from Figure 2(a-d) that the $\mathrm{ZnO}$ NRs with hexagonal faces are well aligned, uniform, highly dense and perpendicular to the substrates as well. The average diameters of NRs for common paper, flexible plastic, textile fabric and aluminum foil are around 100-120 nm, 160-170 nm, 100-110 nm and 170-180 nm, respectively. While the average lengths of the NRs for common paper, flexible plastic, textile fabric and aluminum foil are $\sim 1-1.2 \mu \mathrm{m}, \sim 2 \mu \mathrm{m}, \sim 1.5$ $\mu \mathrm{m}$ and $\sim 2-2.4 \mu \mathrm{m}$, respectively, as shown in Figure $2(\mathrm{a}-\mathrm{d})$. It is already reported that the performance of the nanogenerators was not dependent on the substrates but mainly depended on the crystal quality, effective length, diameter and density of the nanowires across the substrate (Riaz et al. 2011). In our case, the aspect ratio and the density of the $\mathrm{ZnO}$ nanorods (NRs) have influenced the recorded output signals in consistence with other reports (Wang and Song 2006). 
The XRD patterns of the ZnO NRs grown on common paper, flexible plastic, textile fabric and aluminum foil substrates are shown in Figure 3(a-d). All the diffraction peaks can be indexed to the hexagonal wurtzite phase of $\mathrm{ZnO}$ NRs, and the obtained results are consistent with the standard JCPDS card no. 36-1451. However, Figure 3(a-d) shows that the intensity of the 002 peak is varying with respect to the substrate. In Figure 3(a), the XRD pattern of common paper shows that the intensity of 002 peak is relatively less compared with the other substrates. The more intense peak in Figure 3(a) belongs to silver. While in Figure $3(\mathrm{~b}-\mathrm{d})$ the intensity of the 002 peaks is higher compared to the other peaks in the XRD pattern, which gives strong evidence about the preferential growth of $\mathrm{ZnO}$ NRs along the caxis (Yang et al. 2009). The highly intense (002) peak in Figure 3(d) is providing a clear evidence that aluminum foil is more favorable for the growth of $\mathrm{ZnO}$ NRs. While the strongest intensity peak in Figure 3(d) belongs to aluminum foil.

The generation of the piezoelectric potential was described in detail in previously published reports (Wang and Song 2006; Yang et al. 2009). In brief common paper, flexible plastic, textile fabric and aluminum foil were used as substrates to grow $\mathrm{ZnO}$ NRs, and then AFM (having a Si tip coated with a Pt film) was used for the measurement of piezoelectric potential. The schematic diagram in Figure 4 describes the process of electrical pulse generation by ZnO NRs. Figure 4(a) shows the start of the AFM scan process. When the AFM tip scans across the top of the ZnO NRs, the deflection of the ZnO NR created positive and negative potential along the stretched and compressed sides of the NR as shown in Figure 4(b). Moreover, the negative piezoelectric potential sets the Schottky barrier to forward bias; hence, the free electrons can flow across the interface and resulted in a transient current in the direction of the AFM tip. This is happened when the tip touches the compressed side of the NR. The presence of the Schottky barrier forces the current to flow in the direction along which the Schottky barrier is at forward bias. Since we have thousands of NRs that generate 
electricity simultaneously, the Schottky barrier adds all of the generated currents and forces them to flow in the same direction (Hu et al. 2010). Figure 4(c) reveals the position when the NR was released by the AFM tip and it regained its original shape and position. Then, Figure 4(d) shows the re-track of the AFM scan in which the polarization is taking place in the same manner as it takes place in Figure 4(b).

Figure 5(a-d) shows the three-dimensional electrical images, when the AFM tip scanned over the $\mathrm{ZnO}$ NRs arrays covering an area of around $10 \mu \mathrm{m} 2$. In the voltage output image, many sharp output peaks were observed and they were typically about 5-25 times higher than the noise level as shown in Figure 5(a-d). As we know from previous published reports, the deformation of $\mathrm{ZnO}$ NRs produces piezoelectric potential within the NR, which drives the flow of external electrons and produces electric power output. Remember that when the AFM tip started to deflect the ZnO NRs no output potential was exhibited, until the AFM tip achieves its maximum in deflection of NR then output potential was recorded. The obtained results for common paper, flexible plastic, textile fabric and aluminum foil substrates are $16.2 \mathrm{mV}, 23.2 \mathrm{mV}, 38.5 \mathrm{mV}$ and $43.3 \mathrm{mV}$, respectively, as shown in Figure 5(a-d).

For $\mathrm{ZnO} \mathrm{NR}$ that can effectively produce electric power output, the I-V results must always represent Schottky behavior. In principle, the Schottky barrier works as a "gate" that prevents the flow of electrons through the $\mathrm{ZnO} \mathrm{NR}$ and preserves the piezoelectric potential. This also contributes to gathering of electrons, which leads to a higher discharge rate. Whereas the $\mathrm{ZnO} \mathrm{NR}$ works as a "capacitor" in order to store electrons and then drive them back and forth through the external circuit in accordance with the stretch and release of the $\mathrm{ZnO}$ NR. Figure 6(a-d) shows the Schottky behavior for common paper, flexible plastic, textile fabric and aluminum foil substrates, respectively.

Table 1 shows the comparison of the presented work with already published reports on textile fabric, common paper and flexible plastic in order to highlight the improved 
output voltage of these substrates by employing ACG method and to observe the effect of substrate, when growth and measurement conditions remains unchanged.

\section{Conclusions:}

In the present work, we have harvested piezoelectricity from $\mathrm{ZnO}$ NRs using common paper, flexible plastic, textile fabric and aluminum foil as flexible substrates. We conclude that aluminum foil substrate is more favorable for the growth of $\mathrm{ZnO}$ NRs using the ACG method due to the preferential growth of $\mathrm{ZnO}$ NRs along the [0001] direction, and also the fast growth rate along that direction is supported by the aluminum foil substrate in the growth solution. The obtained $\mathrm{ZnO}$ NRs on all the substrates in general and on aluminum foil in particular are highly dense, well aligned and perpendicular to the substrates with good crystal quality. The ZnO NRs produced an output voltage around $16.2 \mathrm{mV}, 38.5 \mathrm{mV}, 23.2 \mathrm{mV}$ and $43.3 \mathrm{mV}$ for common paper, flexible plastic, textile fabric and aluminum foil substrate, respectively. The improvement in the performance of common paper, textile fabric and flexible plastic in comparison with reported values suggests that ACG method more suited to the chemical properties of the flexible substrates. It is believed that by exploiting the flexibility of the substrate the output electricity of the ZnO NRs can be increased due to the possible addition in the flexibility of the fabricated $\mathrm{ZnO}$ NRs. In addition to this, the fabrication of nanogenerators on flexible substrates, more specifically on aluminum foil substrate, can pave the way to develop battery-less devices for future applications.

\section{References:}

Arnold, M. S., P. Avouris, Z. W. Pan, and Z. L. Wang. 2003. "Field-effect transistors based on single semi-conducting oxide nanobelts." The Journal of Physical Chemistry B 107:659-63. 
Bradley, K., J.-C. P. Gabriel, and G. Gruner. 2003. "Flexible nanotube electronics.” Nano Letters 3:1353-5.

Buchine, B. A., W. L. Hughes, F. L. Degertekin, and Z. L. Wang. 2006. "Bulk acoustic resonator based piezoelectric ZnO belts.” Nano Letters 6:1155-9.

Cao, Q., S.-H. Hur, Z. T. Zhu, Y. Sun, C. Wang, M. Meitl, M. Shim, and J. A. Rogers. 2006. "Highly bendable, transparent thin-film transistors that use carbon-nanotube-based conductors and semiconductors with elastomeric dielectrics." Advanced Materials 18:304-09.

Chang, C. J., Y. H. Lee, C. A. Dai, C. C. Hsiao, S. H. Chen, N. P. D. Nurmalasari, J. C. Chen, Y. Y. Cheng, W. P. Shih, and P. Z. Chang. 2011. "A large area biomaterial sheet of piezoelectric nanogenerators for energy harvesting: effect of RF sputtering on ZnO nanorod." Microelectronic Engineering 88:2236-41.

Chik, H., J. Liang, S. G. Cloutier, N. Kouklin, and J. M. Xu. 2004. "Periodic array of uniform $\mathrm{ZnO}$ nanorods by second order self-assembly.” Applied Physics Letters 84:3376-8.

Gao, P. G., J. Song, J. Liu, and Z. L. Wang. 2007. "Nanowire piezoelectric nanogenerators on plastic substrates as flexible power source for nanodevices." Advanced Materials 19:67-72.

Gullapalli, H., V. S. M. Vemuru, A. Kumar, B. M. Andres, R. Vajtai, M. Terrones, S. Nagarajaiah, and P. M. Ajayan. 2010. "Flexible piezoelectric ZnO-paper nanocomposite strain sensor." Small 6:1641-6.

He, J. H., C. L. Hsin, J. Liu, L. J. Chen, and Z. L. Wang. 2007. "Piezoelectric gated diode of a single $\mathrm{ZnO}$ nanowire.” Advanced Materials 19:781-4.

Hsu, C.-L., and K.-C. Chen. 2012. "Improving piezoelectric nanogenerators comprises $\mathrm{ZnO}$ nanowires by bending the flexible PET substrate at low vibration frequency." The Journal of physical chemistry C 116:9351-5. 
Hsueh, T. J., C. L. Hsu, S. J. Chang, and I. C. Chen. 2007. "Laterally grown ZnO nanowires ethanol gas sensors." Sensors and Actuators B 126:473-7.

Hu, Y. F., Y. Zhang, Y. L. Chang, R. L. Snyder, and Z. L. Wang. 2010. “Optimizing the power output of a ZnO photocell by piezopotential." ACS Nano 4:4220-4.

Huang, M. H., S. Mao, H. Feick, H. Yan, Y. Wu, H. Kind, E. Weber, R. Russo, and P. Yang. 2001. "Room-temperature Ultraviolet nanowire nanolasers.” Science 292:1897-9.

Khan, A., M. A. Abbasi, M. Hussain, Z. H. Ibupoto, J. Wissting, O. Nur, and M. Willander. 2012. "Piezoelectric nanogenerators based on zinc oxide nanorods grown on textile cotton fabric.” Applied Physics Letters 101:193506(1-4).

Lao, C. S., Q. Kuang, Z. L. Wang, C. M. Park, and Y. Deng. 2007. "Polymer functionalized piezoelectric-FET as humidity/chemical nanosensors.” Applied Physics Letters 90: $262107(1-3)$.

Law, J. B. K., and J. T. L. Thong. 2006. "Simple fabrication of a $\mathrm{ZnO}$ nanowires photodetector with a fast photoresponse time.” Applied Physics Letters 88:133114(1-3).

Lu, Z. H., J. X. Zhou, A. J. Wang, N. Wang, and X. N. Yang. 2011. "Synthesis of aluminium-doped $\mathrm{ZnO}$ nanocrystals with controllable morphology and enhanced electrical conductivity.” Journal of Materials Chemistry 21:4161-7.

Ogata, K., K. Maejima, S. Fujita, and S. Fujita. 2003. "Growth mode control of ZnO towards nanorod structures or high-quality layered structures by metal-organic vapor phase epitaxy.” Journal of Crystal Growth 248:25-30.

Park, W. I., J. S. Kim, G. C. Yi, M. H. Bae, and H. J. Lee. 2004. "Fabrication and electrical characterization of high performance $\mathrm{ZnO}$ nanorod field-effect transistors." Applied Physics Letters 85:5052-4.

Park, W. I., and G. C. Yi. 2004. "Electroluminescence in n-ZnO nanorod arrays vertically grown on p-GaN.” Advanced Materials 16:87-90. 
Qiu, Y., H. Zhang, L. Hu, D. Yang, L. Wang, B. Wang, J. Ji, G. Liu, X. Liu, J. Lin, et al. 2012. "Flexible piezoelectric nanogenerators based on $\mathrm{ZnO}$ nanorods grown on common paper substrates.” Nanoscale 4:6568-73.

Riaz, M., J. H. Song, O. Nur, Z. L. Wang, and M. Willander. 2011. "Study of the piezoelectric power generation of $\mathrm{ZnO}$ nanowire arrays grown by different methods." Advanced Functional Materials 21:628-33.

Soci, C., A. Zhang, B. Xiang, S. A. Dayeh, D. P. R. Aplin, J. Park, X. Y. Bao, Y. H. Lo, and D. Wang. 2007. “ZnO nanowire UV photodetector with high internal gain.” Nano Letters 7:1003-09.

Soomro, M. Y., I. Hussain, N. Bano, O. Nur, and M. Willander. 2012. "Piezoelectric power generation from zinc oxide nanowires grown on paper substrate.” Physica Status Solidi (RRL) 6:80-2.

Sun, X. W., J. Z. Huang, J. X. Wang, and Z. Xu. 2008. "A ZnO nanorods inorganic/organic heterostructure light-emitting diode emitting at 342nm." Nano Letters $8: 1219-23$.

Tena-Zaera, R., J. Elias, and C. Levy-Clement. 2008. "ZnO nanowire arrays: Optical scattering and sensitization to solar light.” Applied Physics Letters 93:233119(1-3).

Wang, Z. L., and J. H. Song. 2006. "Piezoelectric nanogenerators based on zinc oxide nanowires arrays." Science 312:242-6.

Wang, X. D., J. H. Song, J. Liu, and Z. L. Wang. 2007. "Direct-current nanogenerator driven by ultrasonic waves." Science 316:102-05.

Yang, J. L., S. J. An, W. I. Park, G. -C. Yi, and W. Choi. 2004. "Photocatalysis using $\mathrm{ZnO}$ thin films and nanoneedles grown by metal-organic chemical vapor deposition." Advanced Materials 16:1661-4. 
Yang, B., A. Kumar, H. Zhang, P. Feng, R. S. Katiyar, and Z. Wang. 2009. "Growth of $\mathrm{ZnO}$ nanostructures on metallic and semiconducting substrates by pulsed laser deposition technique.” Journal of Physics D: Applied Physics 42:045415(1-7).

Yang, R. S., Y. Qin, L. M. Dai, and Z. L. Wang. 2009. "Power generation with laterallypackaged piezoelectric fine wires." Nature Nanotechnology 4:34-9.

Zhang, Y., Y. Liu, and Z. L. Wang. 2011. "Fundamental theory of piezotronics." Advanced Materials 23:3004-13.

\section{Figure Captions:}

Figure 1. Schematic diagram showing the current-amplifier response.

Figure 2. The SEM images of $\mathrm{ZnO}$ nanorods grown on different flexible substrates.

(a) Common Paper (b) Plastic (c) Textile Fabric (d) Aluminium foil.

Figure 3. XRD spectra of $\mathrm{ZnO}$ nanorods grown on

(a) Common Paper (b) Plastic (c) Textile Fabric (d) Aluminium foil.

Figure 4. Schematic diagram showing the mechanism of the electrical pulse generation by $\mathrm{ZnO}$

nanorods.

Figure 5. Three-dimensional plot of the output voltage from $\mathrm{ZnO}$ nanorods grown on
(a) Common Paper
(b) Plastic (c)
(c) Textile Fabric (d)
(d) Aluminium foil.

Figure 6. I-V characteristics for
(a) Common Paper (b)
(b) Plastic (c)
(c) Textile Fabric
(d) Aluminium foil.

Table 1. Comparison of published reports with presented work. 
Figure 1.

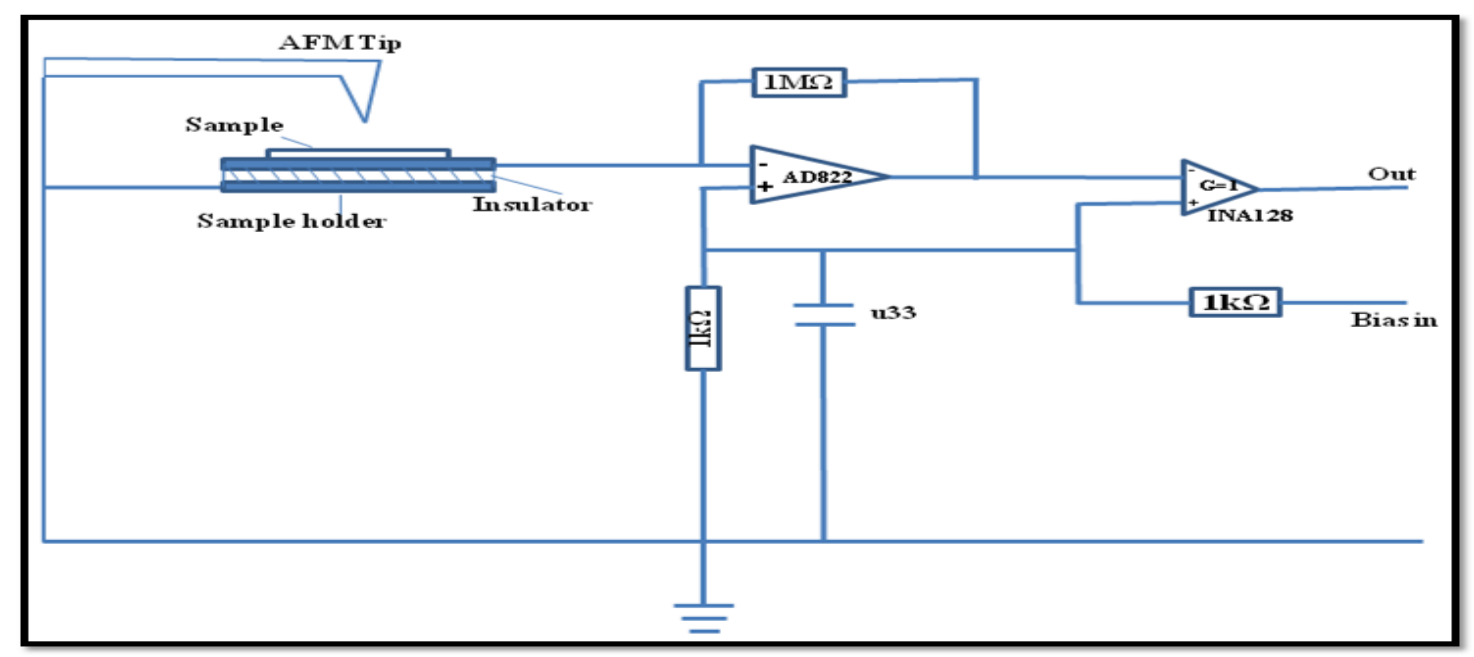

Figure 2.

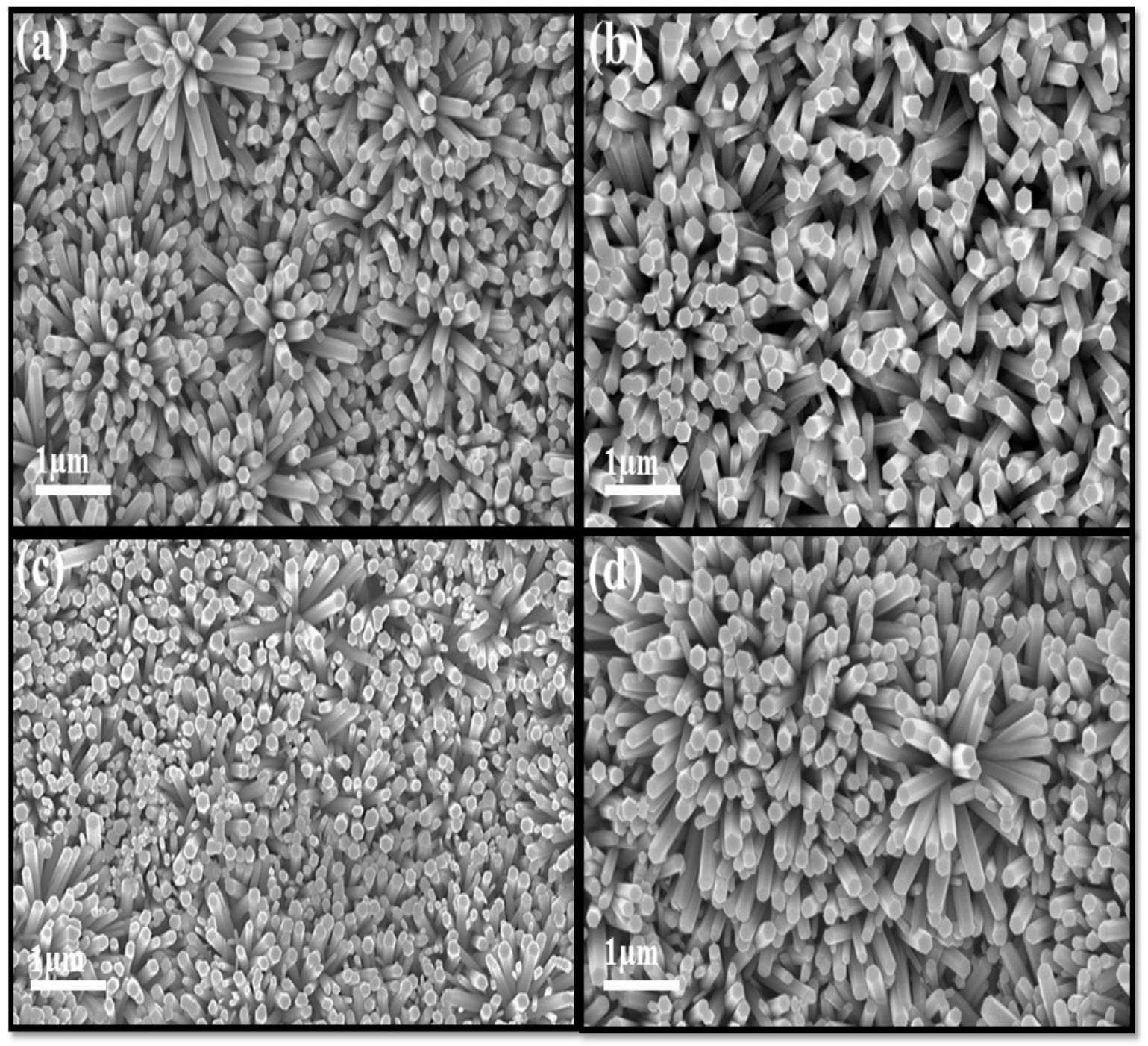


Figure 3.
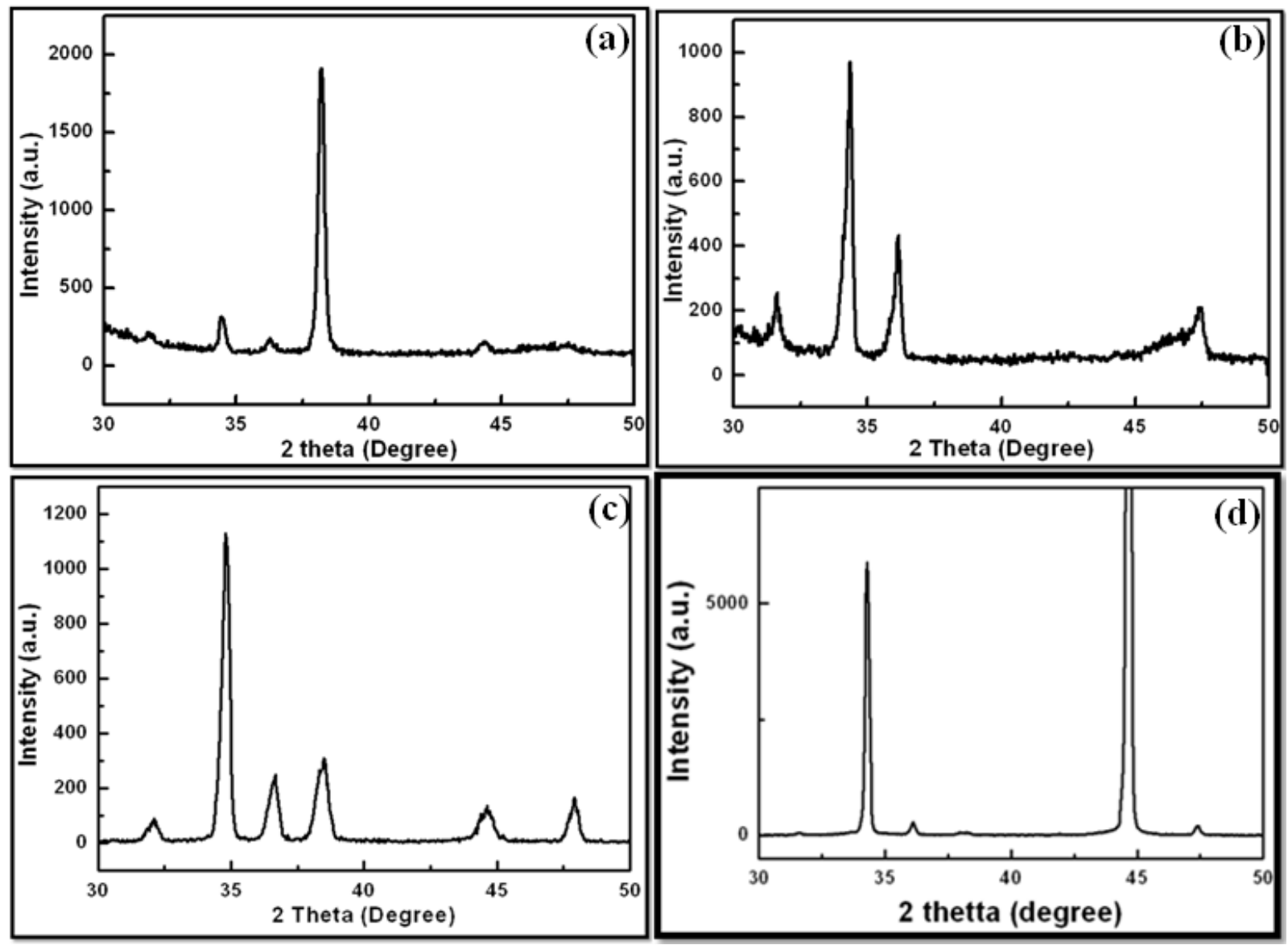

Figure 4.

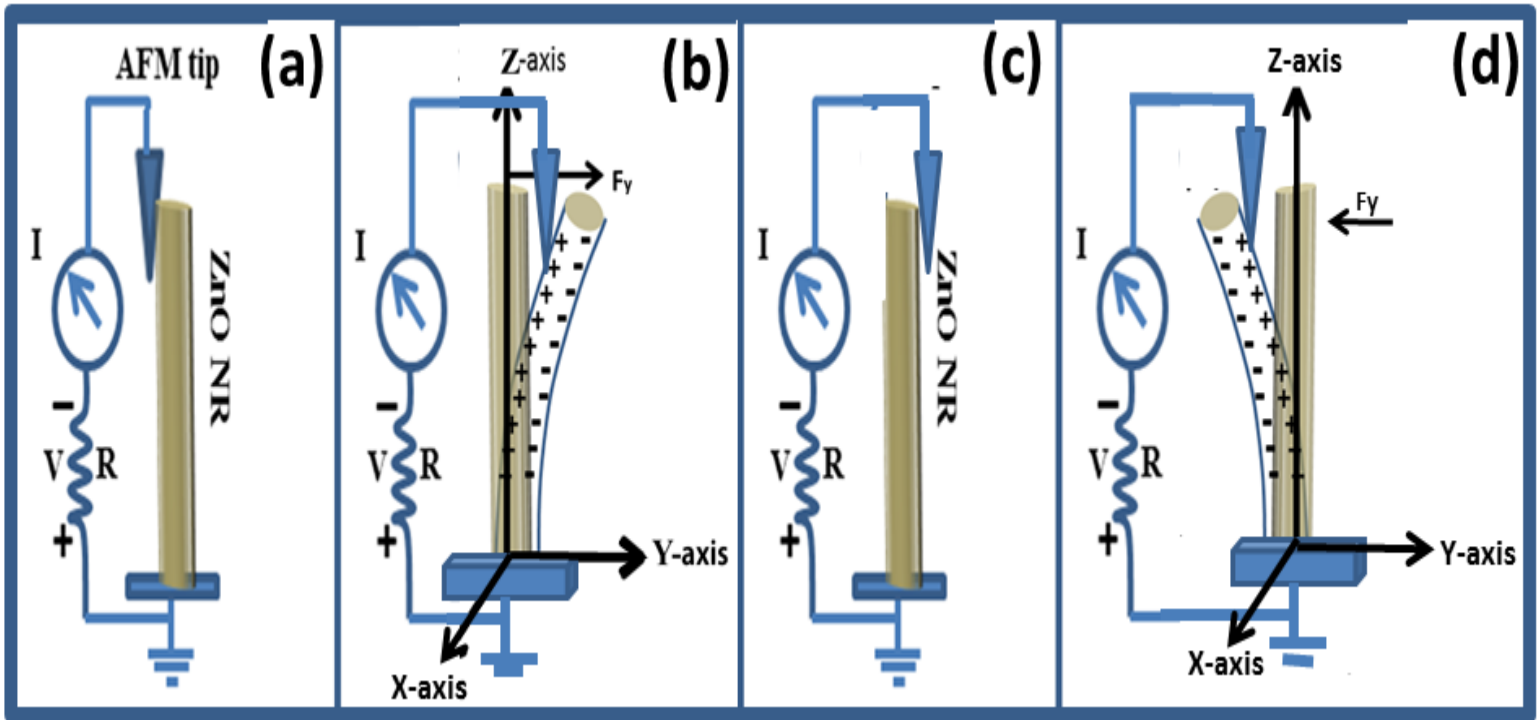


Figure 5.

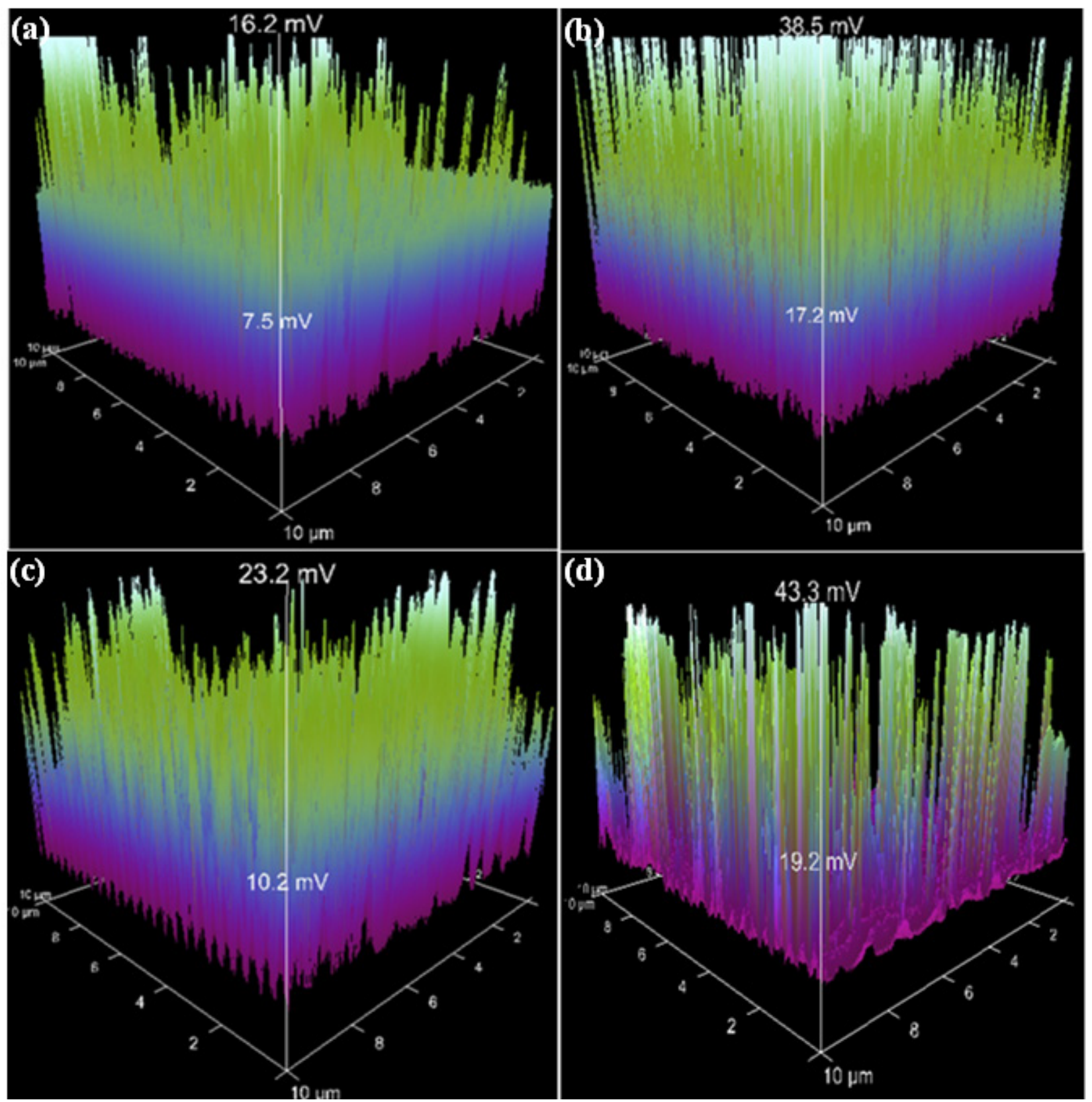


Figure 6.
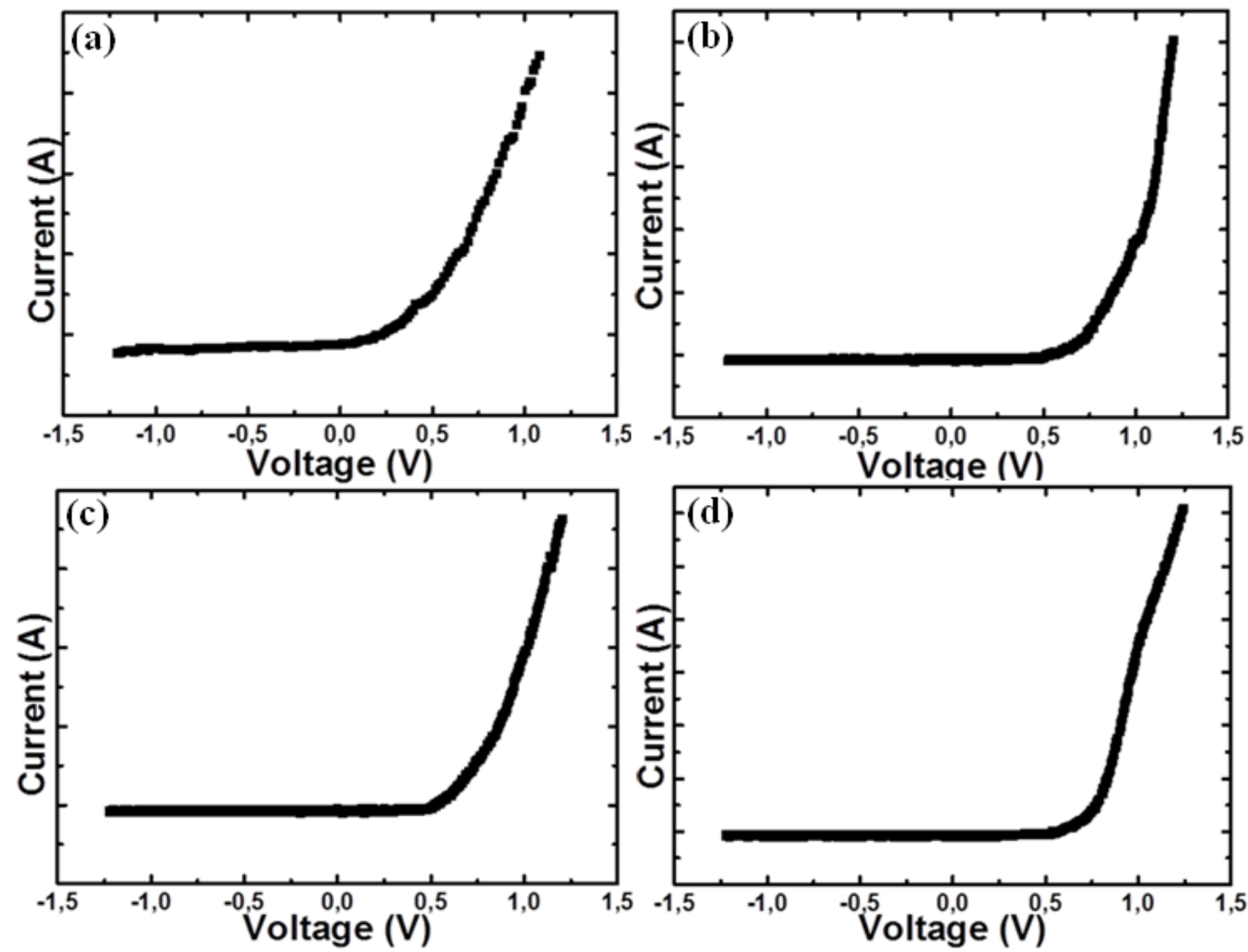

Table 1.

\begin{tabular}{|l|l|l|l|l|}
\hline & Pommon & Plastic & Textile fabric & Aluminium \\
Repoted & 1- $7 \mathrm{mV}[31]$ & $45 \mathrm{mV}$ for single NW, & $9.5 \mathrm{mV}[14]$ & foil \\
work & 2- $10 \mathrm{mV}[23]$ & average $20-25 \mathrm{mV}[24]$ & & \\
\hline Presented & $16.2 \mathrm{mV}$ & $38.5 \mathrm{mV}$ & $23.2 \mathrm{mV}$ & $43.3 \mathrm{mV}$ \\
work & & & & \\
\hline
\end{tabular}

